\title{
Numerical Treatment of Initial Value Problems of Nonlinear Ordinary Differential Equations by Duan-Rach-Wazwaz Modified Adomian Decomposition Method
}

\author{
Ömür Umut*, Serpil Yaşar \\ Department of Mathematics, Faculty of Arts and Sciences, Bolu Abant İzzet Baysal University, Bolu, Turkey \\ Email: *umut_o@ibu.edu.tr, say.maths@gmail.com
}

How to cite this paper: Umut, Ö. and Yaşar, S. (2019) Numerical Treatment of Initial Value Problems of Nonlinear Ordinary Differential Equations by Duan-Rach-Wazwaz Modified Adomian Decomposition Method. International Journal of Modern Nonlinear Theory and Application, 8, 17-39. https://doi.org/10.4236/ijmnta.2019.81002

Received: November 6, 2018

Accepted: January 29, 2019

Published: February 1, 2019

Copyright ( 2019 by author(s) and Scientific Research Publishing Inc. This work is licensed under the Creative Commons Attribution International License (CC BY 4.0).

http://creativecommons.org/licenses/by/4.0/

(c) (i) Open Access

\begin{abstract}
We employ the Duan-Rach-Wazwaz modified Adomian decomposition method for solving initial value problems for the systems of nonlinear ordinary differential equations numerically. In order to confirm practicality, robustness and reliability of the method, we compare the results from the modified Adomian decomposition method with those from the MATHEMATICA solutions and also from the fourth-order Runge Kutta method solutions in some cases. Furthermore, we apply Padé approximants technique to improve the solutions of the modified decomposition method whenever the exact solutions exist.
\end{abstract}

\section{Keywords}

Adomian Decomposition Method, Duan-Rach-Wazwaz Modified Adomian Decomposition Method, Initial Value Problem, Nonlinear Ordinary Differential Equation, Mathematica Solution, 4-th Order Runge Kutta Method, Padé Approximants

\section{Introduction}

Phenomena as diverse as the oscillations of a suspension bridge, the spread of a disease, and the motion of the planets are governed by nonlinear differential equations. Most of these nonlinear equations do not have analytical solutions, so approximation and numerical techniques must be used. The Adomian decomposition method (ADM), introduced by Adomian [1] [2] [3] [4] provides immediate and visible symbolic terms of analytic solutions as well as numerical ap- 
proximate solutions to both linear and nonlinear problems without unphysical restrictive assumptions such as required by linearization, perturbation or discretization [1] [2] [3] [4]. It provides the solution in a rapidly convergent series with easily computable components if the equation has a unique solution. The technique uses a decomposition of the nonlinear operator as a series of Adomian functions. Each term of the series is a generalized polynomial, called the Adomian polynomial. The ADM has been successfully applied to a wide class of problems arising in applied sciences and engineering [1]-[14] over three decades.

Adomian decomposition method has led to a number of modifications made by various researchers for different purposes such as to improve the accuracy, or increase the speed of convergence, or expand the application of the original method. Adomian and Rach [11] introduced modified Adomian polynomials which converge slightly faster than the original polynomials and are convenient for computer generation. Wazwaz [12] [13] used Padé approximants to the solution obtained using a modified decomposition method and found that not only does this improve the results, but also that the error decreases with the increase of the degree of the Padé approximants. The later modifications of ADM were proposed by Wazwaz [14], Wazwaz and El-Sayed [15], Duan [16] [17] [18] [19] [20], Duan and Rach [21] [22] [23], Duan, Rach and Wazwaz [24] [25].

In this paper, we consider the applications of the Duan-Rach-Wazwaz modification of ADM to the initial value problems (IVPs) for the systems of nonlinear ordinary differential equations (ODEs). In 2013, Duan, Rach and Wazwaz [25] presented a reliable modification of the ADM which bases on the previous modification schemes [14]-[24], and computes the solutions of variable coefficients higher-order nonlinear initial value problems (IVPs) and solutions of systems of coupled nonlinear IVPs. To implement these algorithms they also designed multistage decomposition and numeric algorithms, and presented MATHEMATICA routines PSSOL and NSOL.

The text is organized as follows. The basic principles of ADM are given in Section 2. For the numerical solutions of the IVPs for the systems of nonlinear differential ODEs, the frameworks of the Duan-Rach-Wazwaz modification are presented in Section 3. In Section 4, numerical treatments of the nonlinear IVPs using the modified technique and MATHEMATICA numerical solution are performed. The solutions of some problems are also computed by using fourth-order Runge Kutta method (RK4) and the comparisons of the results are presented. A brief conclusion is given in Section 5. All computations are carried out in MATHEMATICA.

\section{Basic Principles of the Adomian Decomposition Method}

Consider the general nonlinear ODE in the Adomian's operator-theoretic form

$$
L u+R u+N u=g(t),
$$

where $g$ is a given analytic function and $u$ is the unknown solution, and $L$ is the 
linear operator to be inverted, $R$ is the linear remainder operator, and $N$ is an analytic nonlinear operator. We remark that the choice of the linear operator is designed to yield an easily invertible operator with resulting trivial integrations. This means that the choice is not unique. Generally we choose $L=\frac{\mathrm{d}^{n}}{\mathrm{~d} t^{n}}(\cdot)$ for $n$-th order ODEs, then its inverse $L^{-1}$ follows as the $n$-fold definite integration operator from $t_{0}$ to $t$. Hence, we have $L^{-1} u=u-\psi$, where $\psi$ is determined using the initial conditions.

Application of $L^{-1}$ to each side of Equation (1) yields

$$
u(t)=\gamma(t)-L^{-1}[R u(t)+N u(t)]
$$

where $\gamma(t)=\psi(t)+L^{-1} g(t)$.

The ADM decomposes the solution into a series

$$
u(t)=\sum_{i=0}^{\infty} u_{i}(t),
$$

and then decomposes the nonlinear term into a series of Adomian polynomials

$$
N u(t)=\sum_{i=0}^{\infty} A_{i}\left(u_{0}(t), \cdots, u_{i}(t)\right),
$$

where $A_{i}, i \geq 0$ are called the Adomian polynomials and generated by the definitional formula

$$
A_{i}=\left.\frac{1}{i !} \frac{\partial^{i}}{\partial \lambda^{i}}\left[f\left(\sum_{k=0}^{\infty} u_{k} \lambda^{k}\right)\right]\right|_{\lambda=0},
$$

where $\lambda$ is a grouping parameter of convenience. The formulas of the first four Adomian polynomials for the one-variable simple analytic nonlinearity $N u(t)=f(u(t))$ are listed as

$$
\begin{gathered}
A_{0}=f\left(u_{0}\right), \\
A_{1}=f^{\prime}\left(u_{0}\right) u_{1}, \\
A_{2}=f^{\prime}\left(u_{0}\right) u_{2}+f^{\prime \prime}\left(u_{0}\right) \frac{u_{1}^{2}}{2 !}, \\
A_{3}=f^{\prime}\left(u_{0}\right) u_{3}+f^{\prime \prime}\left(u_{0}\right) u_{1} u_{2}+f^{(3)}\left(u_{0}\right) \frac{u_{1}^{3}}{3 !}, \\
A_{4}=f^{\prime}\left(u_{0}\right) u_{4}+f^{\prime \prime}\left(u_{0}\right)\left(\frac{u_{2}^{2}}{2 !}+u_{1} u_{3}\right)+f^{(3)}\left(u_{0}\right) \frac{u_{1}^{2} u_{2}}{3 !}+f^{(4)}\left(u_{0}\right) \frac{u_{1}^{4}}{4 !} .
\end{gathered}
$$

In the Duan-Rach-Wazwaz modification, by using Duan's Corollary 3 algorithm [18] the one variable Adomian polynomials are written as

$$
A_{0}=f\left(u_{0}\right), \quad A_{i}=\sum_{k=1}^{i} C_{i}^{k} f^{(k)}\left(u_{0}\right) \text { for } i \geq 1,
$$

where the coefficients $C_{i}^{k}$ are defined recursively [18] as

$$
C_{i}^{1}=u_{i}, i \geq 1, \quad C_{i}^{k}=\frac{1}{i} \sum_{j=0}^{i-k}(j+1) u_{j+1} C_{i-1-j}^{k-1}, 2 \leq k \leq i .
$$


The formulae in (7) does not involve the differentiation operator for the coefficients $C_{i}^{k}$ [20] [21] [22], but requires only addition and multiplication. So, it is more convenient for computer algebra systems.

The definitional formula of the Adomian polynomials for decomposing multivariable nonlinear functions occurring in either single nonlinear $n$ th-order ODEs $f\left(u, u^{\prime}, u^{\prime \prime}, \cdots, u^{(n-1)}\right)$ or in systems of coupled nonlinear ODEs with multivariable nonlinearities are published by Adomian and Rach in [6]. By assuming $f$ is an $m$-ary analytic function $f\left(u_{1}, \cdots, u_{m}\right)$, where the $u_{k}$, for $1 \leq k \leq m$ are the unknown functions to be determined, the solutions $u_{q}, q=1, \cdots, m$ and the nonlinear function $f\left(u_{1}, \cdots, u_{m}\right)$ are decomposed as

$$
u_{q}=\sum_{j=0}^{\infty} u_{q, j}, q=1, \cdots, m \text { and } f\left(u_{1}, \cdots, u_{m}\right)=\sum_{i=0}^{\infty} A_{i},
$$

where the multivariable Adomian polynomials $A_{i}$ depend on the $m(i+1)$ solution components $u_{1,0} u_{1,1} \cdots u_{1, i} ; u_{2,0} u_{2,1} \cdots u_{2, i} ; \cdots ; u_{m, 0} u_{m, 1} \cdots u_{m, i}$ and are defined by [6]

$$
A_{i}=\left.\frac{1}{i !} \frac{\partial^{i}}{\partial \lambda^{i}} f\left(\sum_{j=0}^{\infty} u_{1, j} \lambda^{j}, \cdots, \sum_{j=0}^{\infty} u_{m, j} \lambda^{j}\right)\right|_{\lambda=0},
$$

where $\lambda$ is a grouping parameter of convenience. The first $m$-variable Adomian polynomial $A_{0}$ is $A_{0}=f\left(u_{0}\right)$, where $u_{0}=\left(u_{1,0} \cdots u_{m, 0}\right)$.

Substitution of the Adomian decomposition series for the solution $u(t)$ and the series of Adomian polynomials for the nonlinearity $N u$, Equations (3) and (4) respectively, into Equation (2) yields

$$
\sum_{i=0}^{\infty} u_{i}(t)=\gamma(t)-L^{-1}\left[R \sum_{i=0}^{\infty} u_{i}(t)+\sum_{i=0}^{\infty} A_{i}(t)\right] .
$$

The solution components $u_{i}(t)$ may be determined by one of the several advantageous recursion schemes, which differ from another by the choice of the initial solution component $u_{0}(t)$, beginning with the classic Adomian recursion scheme

$$
u_{0}(t)=\gamma(t), u_{i+1}(t)=-L^{-1}\left[R u_{i}(t)+A_{i}(t)\right], i \geq 0
$$

where Adomian has chosen $u_{0}(t)=\gamma(t)$ as the initial solution. All the solution components $u_{i}(t), i \geq 0$ of the solution $u(t)$ can be determined using Equation (11) and hence, the solution series follows immediately [25]. We remark that the convergence of the Adomian series has already been proved by several investigators [26] [27].

\section{Duan-Rach-Wazwaz Modification of the Adomian Decomposition Method}

We illustrate the general frameworks of the Duan-Rach-Wazwaz modification of the Adomian decomposition method [25] for solving the first-order differential equations and the systems of coupled nonlinear differential equations numerically. Throughout the section we assume the equations are in canonical 
forms.

\subsection{IVP of the First-Order Nonlinear ODE}

We consider the following first-order nonlinear, nonhomogeneous differential equation subject to a bounded initial condition

$$
\begin{gathered}
u^{\prime}(t)+\alpha_{0}(t) u(t)+\beta(t) f(u(t))=g(t), \\
u(0)=C_{0} .
\end{gathered}
$$

We assume that the nonhomogeneous term $g$ and system coefficients $\alpha_{0}(t)$ and $\beta(t)$ are analytic functions.

In Adomian's operator-theoretic form, the Equation (12) can be written as

$$
L u(t)+R u(t)+N u(t)=g(t),
$$

where $L$ is the linear operator, $R$ is the linear remainder operator and $N$ is the nonlinear operator such that

$$
L=\frac{\mathrm{d}}{\mathrm{d} t}(.), \quad R u(t)=\alpha_{0}(t) u(t), \quad N u(t)=\beta(t) f(u(t)) .
$$

We note that, accordance by ([27], page 105) $f(u)$ and $g$ are continuously differentiable at any order and they can be expandable in entire series in the neighbourhood of $t=0$, therefore, sum function $u(t)$ is real analytic and indicated series is absolutely, uniformly convergent. Therefore, the presentation formula for function $u(t)$ is meaningful.

From the assumption on the analyticity of the functions $\alpha_{0}(t), \beta(t)$ and $g(t)$ we write the respective Taylor expansion series as

$$
\begin{gathered}
\alpha_{0}(t)=\sum_{i=0}^{\infty} \alpha_{0, i} t^{i} \quad \alpha_{0, i}=\frac{\alpha_{0}^{(i)}(0)}{i !}, \quad \beta(t)=\sum_{i=0}^{\infty} \beta_{i} t^{i}, \quad \beta_{i}=\frac{\beta^{(i)}(0)}{i !}, \\
g(t)=\sum_{i=0}^{\infty} g_{i} t^{i}, \quad g_{i}=\frac{g^{(i)}(0)}{i !} .
\end{gathered}
$$

Application of the Adomian decomposition series and Adomian polynomials series result

$$
u(t)=\sum_{i=0}^{\infty} u_{i}(t), \quad N u(t)=\sum_{i=0}^{\infty} A_{i}(t), \quad f(u(t))=\sum_{i=0}^{\infty} B_{i}(t),
$$

where $f(u)$ is the simple nonlinearity term and can be any analytic function in $u$ and the corresponding one-variable Adomian polynomials $B_{i}(t)$ have the standard formula [6] [16] [17] [18] $B_{i}(t)=B_{i}\left(u_{0}(t), \cdots, u_{i}(t)\right)$.

By calculating the Cauchy products $\alpha_{0}(t) u(t)$ and $\beta(t) f(u(t))$, respectively we write

$$
\begin{gathered}
R u(t)=\alpha_{0}(t) u(t)=\sum_{i=0}^{\infty} \sum_{m=0}^{i} \alpha_{0, i-m} t^{i-m} u_{i}(t), \\
N u(t)=\beta_{0}(t) f(u(t))=\sum_{i=0}^{\infty} \sum_{m=0}^{i} \beta_{i-m} t^{i-m} B_{i}(t),
\end{gathered}
$$

and hence 


$$
A_{i}(t)=\sum_{m=0}^{i} \beta_{i-m} t^{i-m} B_{i}(t) .
$$

We, next solve Equation (14) for $L u(t)$ and apply the one-fold definite integral operator $L^{-1}=\int_{0}^{t}(.) \mathrm{d} s$ to each side of the resulting equation to get

$$
u(t)=C_{0}+L^{-1} g(t)-L^{-1} R u(t)-L^{-1} N u(t),
$$

since $L^{-1} L u(t)=u(t)-u(0)=u(t)-C_{0}$.

Equation (19) is the equivalent nonlinear Volterra integral equation for the solution $u(t)$.

Evaluating the integrals $L^{-1} g(t), L^{-1} R u(t)$ and $L^{-1} N u(t)$, we write

$$
\begin{gathered}
L^{-1} g(t)=\sum_{i=0}^{\infty} \frac{g_{i} t^{i+1}}{(i+1) !}, \quad L^{-1} R u(t)=\sum_{i=0}^{\infty} \sum_{m=0}^{i} \alpha_{0, i-m} \int_{0}^{t} s^{i-m} u_{m}(s) \mathrm{d} s, \\
L^{-1} N u(t)=\sum_{i=0}^{\infty} \sum_{m=0}^{i} \beta_{i-m} \int_{0}^{t} s^{i-m} B_{m}(s) \mathrm{d} s .
\end{gathered}
$$

By substituting Equations (15), (20) and (21) into Equation (19) we get the Adomian decomposition series as

$$
\begin{aligned}
\sum_{i=0}^{\infty} u_{i}(s)= & C_{0}+\sum_{i=0}^{\infty} \frac{g_{i} t^{i+1}}{(i+1) !}-\sum_{i=0}^{\infty} \sum_{m=0}^{i} \alpha_{0, i-m} \int_{0}^{t} s^{i-m} u_{m}(s) \mathrm{d} s, \\
& -\sum_{i=0}^{\infty} \sum_{m=0}^{i} \beta_{i-m} \int_{0}^{t} s^{i-m} B_{m}(s) \mathrm{d} s .
\end{aligned}
$$

Therefore, the modified recursion scheme is written as

$$
\begin{gathered}
u_{0}(t)=C_{0}, \\
u_{i+1}(t)=\frac{g_{i} t^{i+1}}{(i+1) !}-\sum_{m=0}^{i}\left[\alpha_{0, i-m} u(s)+\beta_{i-m} B_{m}(s)\right] \mathrm{d} s,
\end{gathered}
$$

for $i \geq 0$, where the one-variable Adomian polynomials $B_{m}(t)$ are

$$
B_{m}(t)=B_{m}\left(u_{0}(t), \cdots, u_{m}(t)\right) .
$$

As a result, the $(m+1)$ th-stage solution approximant is given by $\phi_{m+1}(t)=\sum_{i=0}^{m} u_{i}(t)$, for $m \geq 0$, in the limit, it yields the exact solution, that is, $\lim _{m \rightarrow \infty} \phi_{m+1}(t)=\lim _{m \rightarrow \infty} \sum_{i=0}^{m} u_{i}(t)=u(t)$.

By calculating the first several solution components using Equations (22) and (23), we derive the following sequence

$$
\begin{gathered}
u_{0}(t)=C_{0}=c_{0}, \quad u_{1}(t)=\frac{g_{0}-\alpha_{0,0} c_{0}-\beta_{0} B_{0}}{(1)} t=c_{1} t, \\
u_{2}(t)=\frac{g_{1}-\alpha_{0,1} c_{0}-\alpha_{0,0} c_{1}-\beta_{1} B_{0}-\beta_{0} B_{1}}{(2)} t^{2}=c_{2} t^{2}, \cdots,
\end{gathered}
$$

By using induction, we find for $i \geq 0$ that

$$
u_{i+1}(t)=\frac{g_{i}-\sum_{m=0}^{i}\left(\alpha_{0, i-m} c_{m}+\beta_{i-m} B_{m}\right)}{(i+1)} t^{i+1}=c_{i+1} t^{i+1},
$$

where the one-variable Adomian polynomials $B_{m}$ depend solely on the solu- 
tion coefficients $c_{j}$, for $0 \leq j \leq m$, and are determined as

$$
B_{0}=B_{0}\left(c_{0}\right), B_{1}=B_{1}\left(c_{0}, c_{1}\right), B_{2}=B_{2}\left(c_{0}, c_{1}, c_{2}\right), \cdots, B_{m}=B_{m}\left(c_{0}, \cdots, c_{m}\right),
$$

instead of the solution components $u_{j}(t)$ for $0 \leq j \leq m$.

Therefore, we have derived the desired Taylor expansion series for the solution $u(t)$ as $u(t)=\sum_{i=0}^{\infty} c_{i} t^{i}$. By inspection, from the Equation (25), the solution coefficients $c_{i}$ are obtained as the nonlinear recurrence relation

$$
c_{0}=C_{0}, \quad c_{i+1}=\frac{g_{i}-\sum_{m=0}^{i}\left(\alpha_{0, i-m} c_{m}+\beta_{i-m} B_{m}\right)}{(i+1)}, \quad i \geq 0,
$$

where the one-variable Adomian polynomials $B_{m}$ are the same as shown in Equation (26). So, the rule of recursion for the solution coefficients of the first order canonical nonhomogeneous nonlinear IVP with a variable input and variable system coefficients is obtained as $c_{n+1}=c_{n+1}\left(c_{0}, \cdots, c_{i}\right)$, for $n \geq 0$.

\subsection{IVP of the System of Coupled Nonlinear DEs}

We consider the following $n$-th order system of $m$-coupled $n_{k}$-th order nonhomogeneous nonlinear IVPs

$$
\begin{gathered}
u_{k}^{\left(n_{k}\right)}(t)+\sum_{p=1}^{m} \sum_{r=0}^{n_{k}-1} \alpha_{k, p, n_{q}-1-r}(t) u_{p}^{n_{p}-1-r}(t) \\
+\beta_{k}(t) f_{k}\left(u_{1}(t), \cdots, u_{1}^{\left(n_{1}-1\right)}(t) ; \cdots, u_{m}(t), \cdots, u_{m}^{\left(n_{m}-1\right)}(t)\right)=g_{k}(t), \\
u_{k}^{j}(0)=C_{k, j}, 1 \leq k \leq m, 0 \leq j \leq n_{k}-1, m \geq 1, n_{k} \geq 1,
\end{gathered}
$$

where $\alpha_{k, p, j}(t)$ and $\beta_{k}(t)$ for $0 \leq k, p \leq m, 0 \leq j \leq n_{p}-1, n_{k}, n_{p} \geq 1$ are variable system coefficients and $g_{k}(t)$ are variable inputs, and $n=\max \left(n_{1}, n_{2}, \cdots, n_{m}\right), m \geq 1$. We assume that the system coefficients and the system inputs are analytic functions. We also assume that the problem is subject to appropriate $\left(\sum_{p=1}^{m} n_{p}\right)$ bounded initial conditions, i.e., $n_{k}$ bounded initial conditions for each $n_{k}$ th-order nonlinear $\mathrm{DE}, 1 \leq k \leq m$.

In Adomian's operator-theoretic form Equation (27) can written as

$$
L_{k} u_{k}(t)+R_{k} u_{1}(t), \cdots, u_{m}(t)+N_{k} u_{1}(t), \cdots, u_{m}(t)=g_{k}(t),
$$

where $L_{k}$ are the linear operators, $R_{k}$ are the linear remainder operators, i.e., generally sequential-order differential operators, and $N_{k}$ are the nonlinear operators such that

$$
\begin{gathered}
L_{k}=\frac{\mathrm{d}^{n_{k}}}{\mathrm{~d} t^{n_{k}}}(.), \quad R_{k} u_{1}(t), \cdots, u_{m}(t)=\sum_{p=1}^{m} \sum_{r=0}^{n_{p}-1} \alpha_{k, p, n_{p}-1-r}(t) u_{p}^{\left(n_{p}-1-r\right)}(t), \\
N_{k} u_{1}(t), \cdots, u_{m}(t)=\beta_{k}(t) f_{k}\left(u_{1}(t), \cdots, u_{1}^{\left(n_{1}-1\right)}(t) ; \cdots ; u_{m}(t), \cdots, u_{m}^{\left(n_{m}-1\right)}(t)\right) .
\end{gathered}
$$

For a particular $n_{k}$ th-order nonlinear DE in the system represented by Equation (27) or Equation (29), we choose the corresponding solution $u_{k}(t)$ as the primary solution and the solutions $u_{p}(t)$, for $p \neq k$, as the secondary solutions with respect to this same $n_{k}$ th-order DE. We assume that 
$\alpha_{k, p, n_{p}-1}(t), \cdots, \alpha_{k, p, 0}(t), \beta_{k}(t)$ and $g_{k}(t)$ are analytic, and hence have the relating Taylor expansion series

$$
\begin{gathered}
\alpha_{k, p, j}(t)=\sum_{i=0}^{\infty} \alpha_{k, p, j, i} t^{i}, \quad \alpha_{k, p, j, i}=\frac{\alpha_{k, p, j}^{(i)}(0)}{i !}, 1 \leq k, p \leq m, 0 \leq j \leq n_{p}-1, \\
\beta_{k}(t)=\sum_{i=0}^{\infty} \beta_{k, i^{i}}, \quad \beta_{k, i}=\frac{\beta_{k}^{(i)}(0)}{i !} \\
g_{k}(t)=\sum_{i=0}^{\infty} g_{k, i} t^{i}, \quad g_{k, i}=\frac{g_{k}^{(i)}(0)}{i !} .
\end{gathered}
$$

The linear differential operators $L_{k}$ are invertible, and their inverse operators $L_{k}^{-1}$ are given by the $n_{k}$-fold integral $L_{k}^{-1}=\int_{0}^{t} \cdots \int_{0}^{t}(.) \mathrm{d} t \cdots \mathrm{d} t$ for the case of a system of $m$-coupled $n_{k}$ th-order IVPs, where the initial conditions are all specified at the origin.

Application of the Adomian decomposition series and the series of the Adomian polynomials, yields

$$
\begin{gathered}
u_{k}(t)=\sum_{i=0}^{\infty} u_{k, i}(t), \quad N_{k} u_{1}(t), \cdots, u_{m}(t)=\sum_{i=0}^{\infty} A_{k, i}(t), \\
f_{k}\left(u_{1}(t), \cdots, u_{1}^{\left(n_{1}-1\right)}(t) ; \cdots ; u_{m}(t), \cdots, u_{m}^{\left(n_{m}-1\right)}(t)\right)=\sum_{i=0}^{\infty} B_{k, i}(t),
\end{gathered}
$$

where the multi-order differential nonlinearity

$$
f_{k}\left(u_{1}(t), \cdots, u_{1}^{\left(n_{1}-1\right)}(t) ; \cdots ; u_{m}(t), \cdots, u_{m}^{\left(n_{m}-1\right)}(t)\right)=\sum_{i=0}^{\infty} B_{k, i}(t)
$$

can be any analytic function in $u_{1}(t), \cdots, u_{1}^{\left(n_{1}-1\right)}(t) ; \cdots ; u_{m}(t), \cdots, u_{m}^{\left(n_{m}-1\right)}(t)$ and the relating $\sum_{p=1}^{m} n_{p}$-variable Adomian polynomials $B_{k, i}(t)$ have the standard formula [6] [16] [17] [18].

$$
\begin{array}{r}
B_{k, i}(t)=B_{k, i}\left(u_{1,0}(t), \cdots, u_{1, i}(t) ; \cdots ; u_{1,0}^{\left(n_{1}-1\right)}(t), \cdots, u_{1, i}^{\left(n_{1}-1\right)}(t) ; \cdots\right. \\
\left.u_{m, 0}(t), \cdots, u_{m, i}(t) ; \cdots ; u_{m, 0}^{\left(n_{k}-1\right)}(t), \cdots, u_{m, i}^{\left(n_{k}-1\right)}(t)\right)
\end{array}
$$

or equivalently,

$$
\begin{array}{r}
B_{k, i}(t)=B_{k, i}\left(u_{1,0}(t), \cdots, u_{1, i}(t) ; \cdots ; \frac{\mathrm{d}^{n_{1}-1}}{\mathrm{~d} t^{n_{1}-1}} u_{1, n_{1}-1}(t), \cdots, \frac{\mathrm{d}^{n_{1}-1}}{\mathrm{~d} t^{n_{1}-1}} u_{1, i+n_{1}-1}(t) ; \cdots ;\right. \\
\left.u_{m, 0}(t), \cdots, u_{m, i}(t) ; \cdots ; \frac{\mathrm{d}^{n_{k}-1}}{\mathrm{~d} t^{n_{k}-1}} u_{m, n_{k}-1}(t), \cdots, \frac{\mathrm{d}^{n_{k}-1}}{\mathrm{~d} t^{n_{k}-1}} u_{m, i+n_{k}-1}(t)\right)
\end{array}
$$

The relating Cauchy products are

$$
R_{k} u_{1}(t), \cdots, u_{m}(t)=\sum_{p=1}^{m} \sum_{r=0}^{n_{p}-1} \alpha_{k, p, n_{p}-1-r}(t) u_{p}^{\left(n_{p}-1-r\right)}(t)
$$

where

$$
\alpha_{k, p, j}(t) u_{p}^{j}(t)=\sum_{i=0}^{\infty} \alpha_{k, p, t} t^{i} \sum_{s=0}^{\infty} u_{p, s}^{(j)}(t)=\sum_{i=0}^{\infty} \sum_{s=0}^{i} \alpha_{k, p, j, i-s} t^{i-s} \frac{\mathrm{d}^{j}}{\mathrm{~d} t^{j}} u_{p, s+j}(t),
$$

where $j=n_{p}-1-r$, 


$$
\begin{aligned}
N_{k} u_{1}(t), \cdots, u_{m}(t) & =\sum_{i=0}^{\infty} \beta_{k, i} t^{i} \sum_{p=0}^{\infty} B_{k, s}(t) \\
& =\sum_{i=0}^{\infty} \sum_{s=0}^{i} \beta_{k, i-s} t^{i-s} B_{k, s}(t),
\end{aligned}
$$

hence it is also determined that

$$
A_{k, i}(t)=\sum_{i=0}^{\infty} \beta_{k, i} t^{i} B_{k, s}(t)
$$

Next we solve Equation (29) for $L_{k}(t)$ as

$$
L_{k} u_{k}(t)=g_{k}(t)-R_{k} u_{1}(t), \cdots, u_{m}(t)-N_{k} u_{1}(t), \cdots, u_{m}(t)
$$

Applying the $n_{k}$-fold integral operator $L_{k}^{-1}$ to each side of Equation (32), we obtain

$$
L_{k}^{-1} L_{k} u_{k}(t)=L_{k}^{-1} g_{k}(t)-L_{k}^{-1} R_{k} u_{1}(t), \cdots, u_{m}(t)-L_{k}^{-1} N_{k} u_{1}(t), \cdots, u_{m}(t)
$$

By integrating left side of Equation (33) and substituting the values specified in Equation (28) we obtain

$$
L_{k}^{-1} L_{k} u_{k}(t)=u_{k}(t)-\sum_{r=0}^{n_{k}-1} \frac{t^{r}}{r !} C_{k, r} .
$$

Substituting this on the left side of Equation (33), we obtain

$$
u_{k}(t)=\sum_{r=0}^{n_{k}-1} \frac{t^{r}}{r !} C_{k, r}+L_{k}^{-1} g_{k}(t)-L_{k}^{-1} R_{k} u_{1}(t), \cdots, u_{m}(t)-L_{k}^{-1} N_{k} u_{1}(t), \cdots, u_{m}(t) \text {. }
$$

Formula (35) is the equivalent system of $m$-coupled nonlinear Volterra integral equations.

Evaluating the relating integrals, we get

$$
\begin{gathered}
L_{k}^{-1} g_{k}(t)=\sum_{i=0}^{\infty} \frac{g_{k, i} t^{i+n_{k}}}{(i+1) \cdots\left(i+n_{k}\right)}, \\
L_{k}^{-1} R_{k} u_{1}(t), \cdots, u_{m}(t)=\sum_{p=1}^{m} \sum_{r=0}^{n_{p}-1} L_{k}^{-1} \alpha_{k, p, n_{p}-1-r}(t) u_{p}^{\left(n_{p}-1-r\right)}(t),
\end{gathered}
$$

where

$$
L_{k}^{-1} \alpha_{k, p, j}(t) u_{p}^{(j)}(t)=\sum_{i=0}^{\infty} \sum_{s=0}^{i} \alpha_{k, p, j, i-s} L_{k}^{-1} t^{i-s} \frac{\mathrm{d}^{j}}{\mathrm{~d} t^{j}} u_{p, s+j}(t),
$$

where $j=n_{p}-1-r$,

$$
L_{k}^{-1} N_{k} u_{1}(t), \cdots, u_{m}(t)=\sum_{i=0}^{\infty} \sum_{s=0}^{i} \beta_{k, i-s} L_{k}^{-1} t^{i-s} B_{k, s}(t) .
$$

Substitution of the Equations (30), (36), (37), (38) and (39) into Equation (35) yields the following system of $m$-coupled modified recursion schemes

$$
\begin{aligned}
& u_{k, 0}(t)=C_{k, 0}, u_{k, 1}(t)=t C_{k, 1}, u_{k, 2}(t)=\frac{t^{2}}{2 !} C_{k, 2}, \cdots, \\
& u_{k, n_{k}-1}(t)=\frac{t^{n_{k}-1}}{\left(n_{k}-1\right) !} C_{k, n_{k}-1}, \cdots
\end{aligned}
$$




$$
\begin{aligned}
u_{k, i+n_{k}}(t)= & \frac{t^{i+n_{k}}}{\prod_{q=1}^{n_{k}}(i+q)}-\sum_{s=0}^{i} \beta_{k, i-s} L_{k}^{-1} t^{i-s} B_{k, s}(t) \\
& \times \sum_{s=0}^{i} \sum_{p=1}^{m} \sum_{r=0}^{i} \alpha_{k, p, n_{p}-1-r, i-s} L_{k}^{-1} t^{i-s} \frac{\mathrm{d}^{n_{p}-1-r}}{\mathrm{~d} t^{n_{p}-1-r}} u_{p, s+n_{p}-1-r}(t)
\end{aligned}
$$

for $1 \leq k \leq m, n_{k}, n_{p} \geq 1, i \geq 0$.

Therefore, the $(s+1)$ th-stage solution approximants $\Phi_{k, s+1}(t)$ are given by $\Phi_{k, s+1}(t)=\sum_{i=0}^{s} u_{k, i}(t)$ for $s \geq 0$.

From the calculation of the first several solution components, we deduce the following sequence

$$
\begin{aligned}
& u_{k, 0}(t)=C_{k, 0}=c_{k, 0}, u_{k, 1}(t)=t C_{k, 1}=c_{k, 1} t, \cdots, u_{k, n_{k}-1}(t)=\frac{t^{n_{k}-1}}{\left(n_{k}-1\right) !}=c_{k, n_{k}-1} t^{n_{k}-1} \\
& u_{k, i+n_{k}}(t) \\
& =\frac{g_{k, i}-\sum_{s=0}^{i}\left\{\beta_{k, i-s} B_{k, s}+\sum_{p=1}^{m} \sum_{r=0}^{n_{p}-1}\left[\prod_{j=1}^{n_{p}-1-r}(s+j)\right] \alpha_{k, p, n_{p}-1-r, i-s} c_{p, s+n_{p}-1-r}\right\}}{\prod_{q=1}^{n_{k}}(i+q)} t^{i+n_{k}} \\
& =c_{k, n_{k}} t^{i+n_{k}}, i \geq 0
\end{aligned}
$$

where the $\left(\sum_{p=1}^{m} n_{p}\right)$-variable Adomian polynomials $B_{k, s}$ are now constants and depend merely on the solution coefficients $c_{k, j}$ for $0 \leq j \leq n_{p}-1$. They are determined by induction as

$$
\begin{array}{r}
B_{k, s}(t)=B_{k, s}\left(c_{1,0}, \cdots, c_{1, s} ; \cdots ; c_{1,0}^{\left(n_{1}-1\right)}, \cdots, c_{1, s}^{\left(p_{1}-1\right)} ; \cdots ;\right. \\
\left.c_{m, 0}, \cdots, c_{m, s} ; \cdots ; c_{m, 0}^{\left(n_{m}-1\right)}, \cdots, c_{m, s}^{\left(n_{m}-1\right)}\right)
\end{array}
$$

or equivalently,

$$
\begin{array}{r}
B_{k, s}=B_{k, s}\left(c_{1,0}, \cdots, c_{1, s} ; \cdots ;\left\{\prod_{j=1}^{n_{1}-1}(j)\right\} c_{1, n_{1}-1}, \cdots,\left\{\prod_{j=1}^{n_{1}-1}(s+j)\right\} c_{1, s+n_{1}-1} ; \cdots ;\right. \\
\left.c_{m, 0}, \cdots, c_{m, s} ;\left\{\prod_{j=1}^{n_{m}-1}(j)\right\} c_{m, n_{m}-1}, \cdots,\left\{\prod_{j=1}^{n_{m}-1}(s+j)\right\} c_{m, s+n_{m}-1}\right)
\end{array}
$$

instead of the solution components $u_{k, j}(t)$ and solution derivative components $u_{k, j}^{(i)}(t)$ for $1 \leq k \leq m, 0 \leq j \leq s$ and $0 \leq q \leq n_{p}-1$. Thus we have derived the desired Taylor expansion series for each of the $m$ solutions $u_{k}(t)$ as $u_{k}(t)=\sum_{i=0}^{\infty} c_{k, i} t^{i}$ where the solution coefficients $c_{k, i}$ are given by the system of $m$-coupled nonlinear recurrence relations, obtained from inspection of Equation (39), as

$$
\begin{gathered}
c_{k, j}=\frac{c_{k, j}}{j !}, 1 \leq k \leq m, 0 \leq j \leq n_{p}-1, \\
c_{k, i+n_{k}}=\frac{g_{k, i}-\sum_{s=0}^{i}\left\{\beta_{k, i-s} B_{k, s}+\sum_{p=1}^{m} \sum_{r=0}^{n_{p}-1}\left[\prod_{j=1}^{n_{p}-1-r}(s+j)\right] \alpha_{k, p, n_{p}-1-r, i-s} c_{p, s+n_{p}-1-r}\right\}}{\prod_{q=1}^{n_{k}}(i+q)},
\end{gathered}
$$


$i \geq 0, m \geq 1$ and $n_{k} \geq 1$, where the $\left(\sum_{p=1}^{m} n_{p}\right)$-variable Adomian polynomials $B_{k, s}$ depend on the solution coefficients $c_{k, j}$, for $0 \leq j \leq n_{p}-1$, as in Equation (41). Consequently, the rule of recursion for the solution coefficients of the canonical $n$ th-order system of $m$-coupled $n_{k}$ th order nonhomogeneous nonlinear IVPs with variable inputs and variable system coefficients are given as

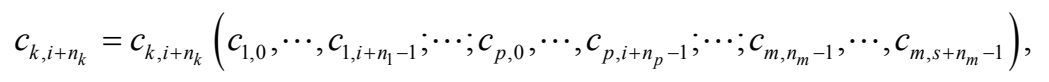

for $i \geq 0,1 \leq k, p \leq m$.

\section{Examples}

In this section, we consider several examples of IVPs for the systems of nonlinear ODEs, which have either quadratic or cubic nonlinearities but, exhibit rather complex behavior. The modified numeric solutions of the problems are obtained by using MATHEMATICA routines PSSOL and NSOL [25]. To compare the results, we have calculated the MATHEMATICA numeric solutions for the systems of differential equations by using the command "NDSolve". We also compute numerical solutions using RK4 in examples 1, 2 and 3. Moreover, we use diagonal Padé approximants [28] [29] [30] to improve the modified results and compute errors in the approximations in the examples 2 and 3 since the exact solutions of these problems are known.

Example 1. Consider the Abel differential equation of the first kind in canonical form. It is a first order, nonhomogeneous differential equation with a cubic nonlinearity [31].

$$
y^{\prime}=\sin t-y^{3}, \quad y(0)=\frac{1}{2},
$$

over the interval $0 \leq t \leq 40$. This nonlinear IVP does not have an exact solution but, a detailed qualitative analysis can be found in [31].

Running PSSOL by taking $n=4$ to output 5th-degree or equivalently 6-term approximation to the solution as

$$
y_{5}(t)=\frac{1}{2}-\frac{t}{8}+\frac{35}{64} t^{2}-\frac{37}{256} t^{3}+\frac{457}{12288} t^{4}-\frac{1823}{16384} t^{5} .
$$

We note that the order of approximation is $O\left(t^{5}\right)$.

Running NSOL for $n=4$ and step size $h=0.05$ to output the numeric solution $y_{5}$ of order 5 which is depicted with red line as the curve of 5th order approximation $y_{5}$ and parametric plot on the left in Figure 1.

As the comparison, MATHEMATICA numeric solution and RK4 solution are found and the curves and the parametric plots of the results are sketched with blue and black lines in the middle and on the right, respectively in Figure 1.

From Figure 1, we conclude that this problem has a limit cycle.

Example 2. Consider the first-order nonhomogeneous nonlinear differential equation with a quadratic nonlinearity [32]

$$
y^{\prime}=y^{2}+2 y+2, \quad y(0)=0,
$$




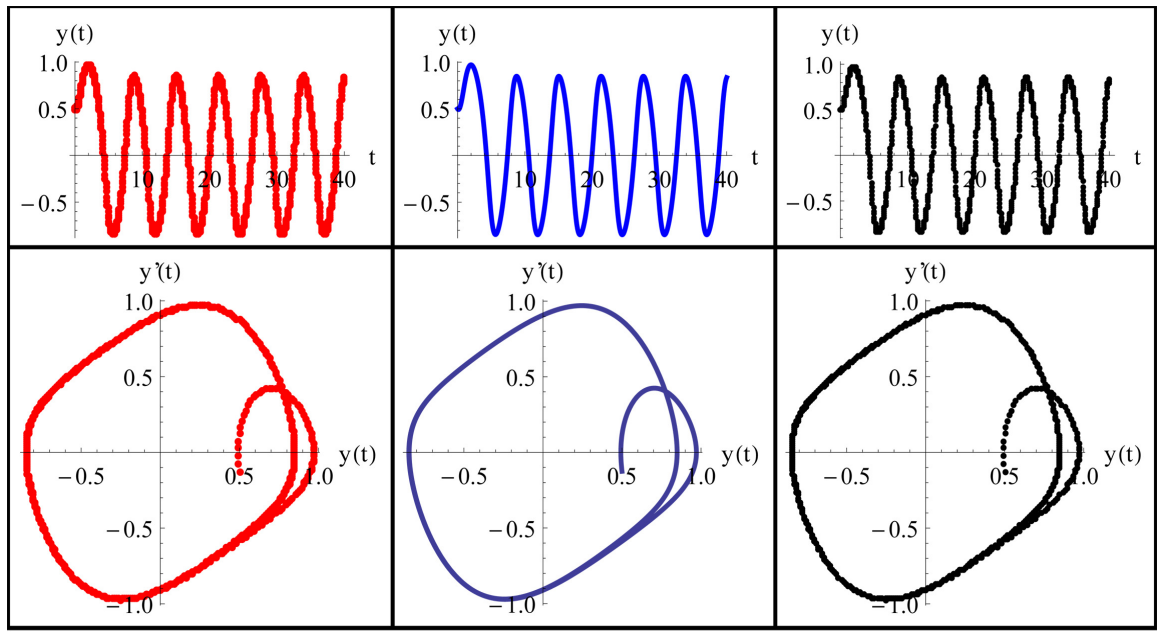

Figure 1. The curves and parametric plots of the 5th-degree MADM approximate solution (red), MATHEMATICA numeric solution (blue) and RK4 solution (black) using the step-size $h=0.05$ over the interval $0 \leq t \leq 40$.

on the interval $0 \leq t \leq \pi / 6$. It has the exact solution $y(t)=-1+\tan \left(t+\frac{\pi}{4}\right)$.

Running PSSOL routine for $n=6,7$ and 8 to output the 13-term, 15-term and 17-term approximants of the solution, respectively, as

$$
\begin{aligned}
y_{12}(t)= & 2 t+2 t^{2}+\frac{8}{3} t^{3}+\frac{10}{3} t^{4}+\frac{64}{15} t^{5}+\frac{244}{45} t^{6}+\frac{2176}{315} t^{7}+\frac{554}{64} t^{8} \\
& +\frac{31744}{2835} t^{9}+\frac{202084}{13175} t^{10}+\frac{2830336}{155925} t^{11}+\frac{2162212}{93555} t^{12} \\
y_{14}(t)= & 2 t+2 t^{2}+\frac{8}{3} t^{3}+\frac{10}{3} t^{4}+\frac{64}{15} t^{5}+\frac{244}{45} t^{6}+\frac{2176}{315} t^{7}+\frac{554}{64} t^{8} \\
& +\frac{31744}{2835} t^{9}+\frac{202084}{13175} t^{10}+\frac{2830336}{155925} t^{11}+\frac{2162212}{93555} t^{12} \\
& +\frac{178946048}{6081075} t^{13}+\frac{1594887848}{42567525} t^{14} \\
y_{16}(t)= & 2 t+2 t^{2}+\frac{8}{3} t^{3}+\frac{10}{3} t^{4}+\frac{64}{15} t^{5}+\frac{244}{45} t^{6}+\frac{2176}{315} t^{7}+\frac{554}{64} t^{8} \\
& +\frac{31744}{2835} t^{9}+\frac{202084}{13175} t^{10}+\frac{2830336}{155925} t^{11}+\frac{2162212}{93555} t^{12} \\
& +\frac{178946048}{6081075} t^{13}+\frac{1594887848}{42567525} t^{14}
\end{aligned}
$$

Indeed, these are the first 12,14 and 16 terms of the Taylor series of the function $y(t)=-1+\tan \left(t+\frac{\pi}{4}\right)$ about the point $t=0$, respectively. So, if it is possible to compute all terms of the series we shall see that the Adomian series for this problem is simply that Taylor series. All terms of the series are positive so, absolute convergence is simply the convergence of the series.

$$
\text { Since }\left|-1+\tan \left(t+\frac{\pi}{4}\right)\right| \leq 1+\frac{\sqrt{3}}{2} \text { on the interval } 0 \leq t \leq \frac{\pi}{6} \text {, and }
$$


$\left|f^{(n)}(0)\right|=\left|\frac{\mathrm{d}^{n}}{\mathrm{~d} y^{n}}\left(y^{2}\right)\right|_{y=0} \mid \leq 2$ for any $n$, accordance by ([26], page 105) the respective truncation errors are $\tau=\frac{2\left(1+\frac{\sqrt{3}}{2}\right)^{12}}{12 !}=7.44224 \times 10^{-6}$, $\tau=\frac{2\left(1+\frac{\sqrt{3}}{2}\right)^{14}}{14 !}=1.42386 \times 10^{-7}$ and $\tau=\frac{2\left(1+\frac{\sqrt{3}}{2}\right)^{16}}{16 !}=2.06581 \times 10^{-8}$.

We note that in these computations approximation orders are $O\left(t^{12}\right), O\left(t^{14}\right)$ and $O\left(t^{16}\right)$, respectively.

The curves of the computed approximants and the exact solution are plotted in Figure 2(a).

The MATHEMATICA command PadeApproximant $\left[y_{2 n+1}(t),\{t, 0,\{n, n\}\}\right]$ for $n=6,7$ and 8 output the [6/6], [7/7] and [8/8] diagonal Padé-approximants of the 13-term, 15-term and 17-term approximants, generated by the routine PSSOL respectively. The curves of the Padé approximants and the exact solution are plotted in Figure 2(b).

Running NSOL routine for $n=11,13$, and 15 and step size $h=0.05$ to generate numeric solutions on the interval $0 \leq t \leq \pi / 6$. In Figure 2(c), the curves of these NSOL numeric solutions and the exact solution are depicted.

As a comparison RK4 solution is computed and depicted with exact solution in Figure 2(d).

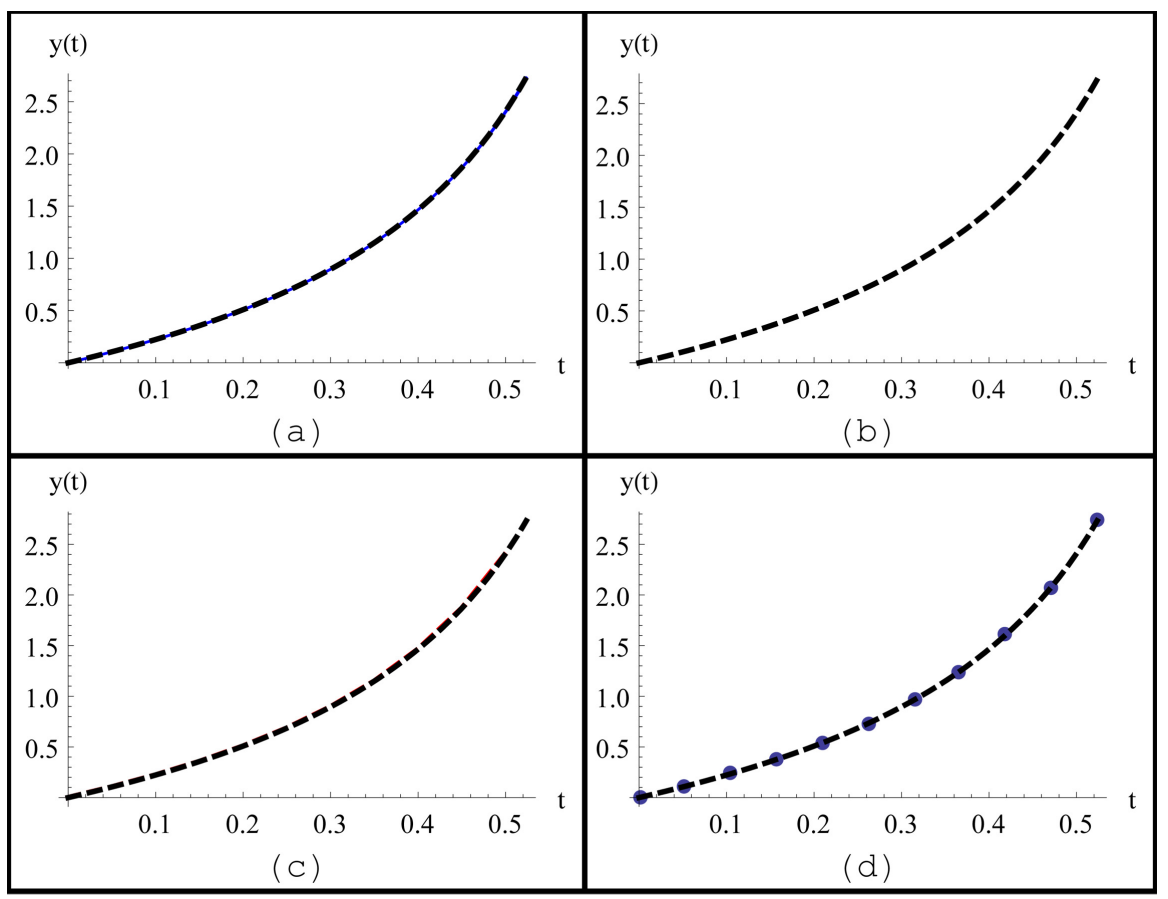

Figure 2. PSSOL outputs and exact solution in (a), Padé-approximants and exact solution in (b), outputs of the NSOL routine and exact solution in (c) and RK4 results and exact solution in (d) for $n=6,7$ and 8 . 
We denote $P_{n}(t)=[n / n]\left\{y_{2 n+1}(t)\right\}$ and consider the absolute error function $\left|E_{n}(t)\right|=\left|P_{n}(t)-y(t)\right|$ for $P_{n}(t)$ on the interval $0 \leq t \leq \pi / 6$ and the maximal error parameter $M E_{n}=\max _{0 \leq t \leq \pi / 6}\left|E_{n}(t)\right|$ for $n=6,7$, and 8 .

In Figure 3, the curves of the absolute error function $\left|E_{n}(t)\right|$ for $n=6,7$, and 8 are given.

From Figure 3 we can conclude that the ADM can be combined with the diagonal Padé approximants to estimate the blow-up time [28]. Since $t=\pi / 4$ is the blow-up time for this problem, this can be seen from the figure.

The maximal error parameters $M E_{n}$ for $n=6,7$ and 8 are given in Table 1.

Table 1 shows that the maximal errors for the exact solution decrease approximately at an exponential rate.

Example 3. Consider the 2-dimensional system of nonlinear differential equations with quadratic nonlinearity [32]

$$
x^{\prime}=x y, y^{\prime}=x^{2}, x(0)=1, y(0)=0,
$$

over the interval $0 \leq t \leq \frac{\pi}{3}$. The exact solutions are $x(t)=\sec t, y(t)=\tan t$.

Running PSSOL for $n=10$ to generate 11-term approximants $x_{11}$ and $y_{11}$ of the solutions $x(t)$ and $y(t)$, respectively as

$$
\begin{aligned}
& x_{11}(t)=1+\frac{t^{2}}{2}+\frac{5 t^{4}}{24}+\frac{61 t^{6}}{720}+\frac{277 t^{8}}{8064}+\frac{50521 t^{10}}{3628800}, \text { and } \\
& y_{11}(t)=t+\frac{t^{3}}{3}+\frac{2 t^{5}}{15}+\frac{17 t^{7}}{315}+\frac{62 t^{9}}{2835}+\frac{1382 t^{11}}{155925} .
\end{aligned}
$$

We note that the respective orders of the approximation are $O\left(t^{10}\right)$ and

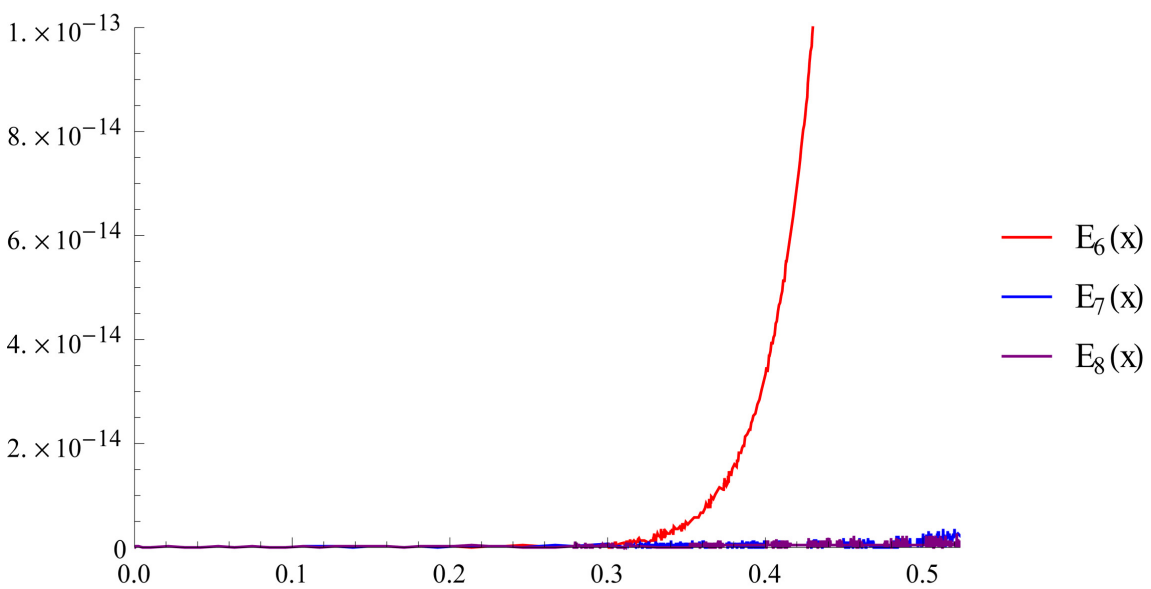

Figure 3. The absolute error function for $n=6$ (Red line), $n=7$ (Blue line) and $n=8$ (Purple line).

Table 1. The maximal error parameters $M E_{n}$ for $n=6,7$ and 8 .

\begin{tabular}{cccc}
\hline$n$ & 6 & 7 & 8 \\
\hline$M E_{n}$ & $1.0747 \times 10^{-12}$ & $1.33227 \times 10^{-15}$ & $8.88178 \times 10^{-16}$ \\
\hline
\end{tabular}


$O\left(t^{11}\right)$

The MATHEMATICA command PadeApproximant $\left[x_{11}(t),\{t, 0,\{5,5\}\}\right]$ and the MATHEMATICA command PadeApproximant $\left[y_{11}(t),\{t, 0,\{5,5\}\}\right]$ for $n=5$ output [5/5] Padé approximants for $x_{11}(t)$ and $y_{11}(t)$ as

$$
\text { Pade } x[5 / 5]=\frac{1+\frac{11 t^{2}}{252}+\frac{13 t^{4}}{15120}}{1-\frac{115 t^{2}}{252}+\frac{313 t^{4}}{15120}} \text {, and Pade } y[5 / 5]=\frac{t-\frac{t^{3}}{9}+\frac{t^{5}}{945}}{1-\frac{4 t^{2}}{9}+\frac{t^{4}}{63}} \text {. }
$$

The outputs of the routines PSSOL, NSOL and RK4 for $n=10,12$ and 14 and correspondingly the outputs of the MATHEMATICA command PadeApproximant for $n=5,6$ and 7 together with the exact solutions $x$ and $y$ are depicted in Figures $4(\mathrm{a})-(\mathrm{c})$.

In Figure 5, the absolute errors for the exact solutions $x(t)$ and $y(t)$ are sketched in (a) and (b), respectively.

We list the maximal error parameters $M E_{n}$ for $n=5,6$ and 7 in Table 2.

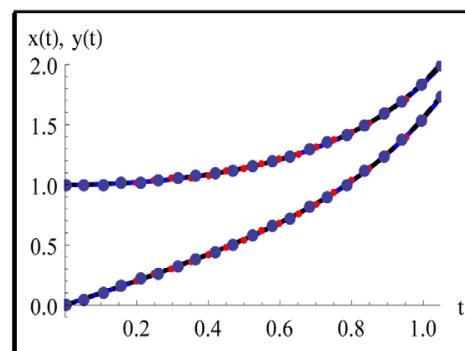

(a)

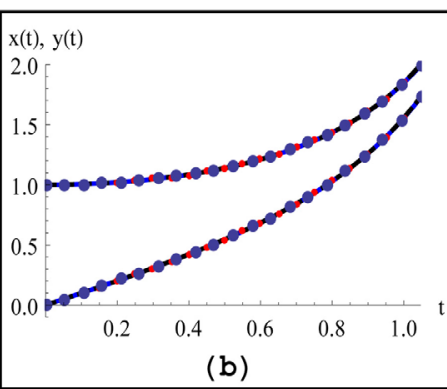

(b)

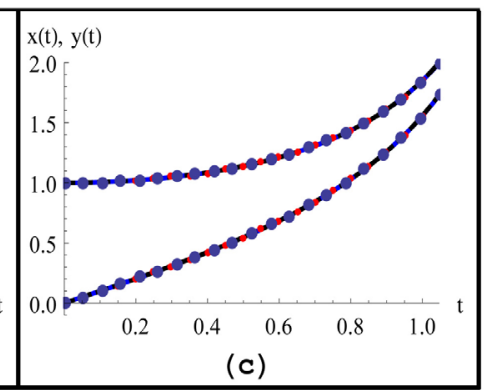

PSSOL, solid

PAD", dashed

- NSOL, dotted

- EXACT, dashed

- RK4, dotted

Figure 4. The curves of PSSOL, NSOL, RK4 outputs and exact solution for $n=5,6$ and 7 in (a), (b) and (c), respectively.

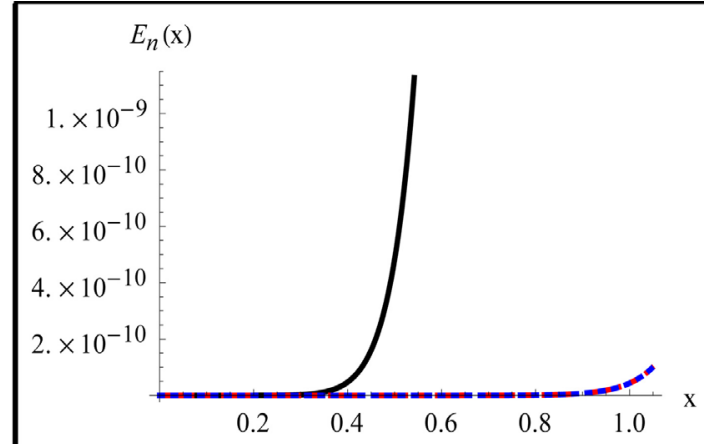

(a)

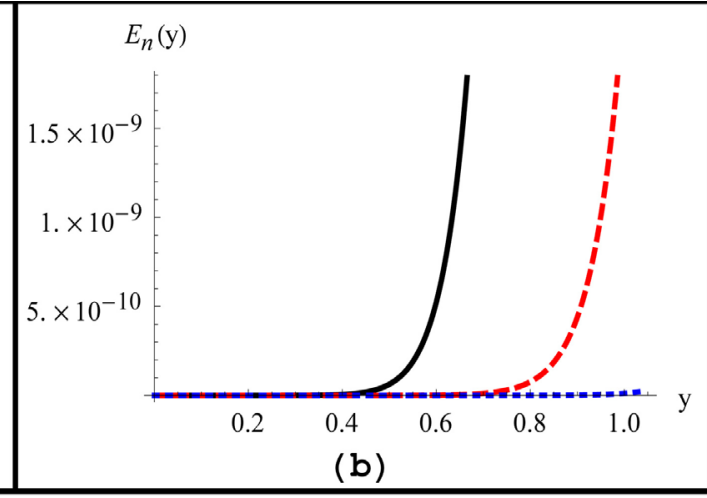

(b)

Figure 5. (a) The absolute error for the $x$-component of the solution, $x(t)=\sec t$; (b) The absolute error for the $y$-component of the solution, $y(t)=\tan t$.

Table 2. The maximal error parameters $M E_{n}$ for $n=5,6$ and 7 .

\begin{tabular}{cccc}
\hline$n$ & 5 & 6 & 7 \\
\hline$M E_{n}(x)$ & $1.23274 \times 10^{6}$ & $4.35763 \times 10^{11}$ & $4.35763 \times 10^{11}$ \\
$M E_{n}(y)$ & $3.17247 \times 10^{7}$ & $2.25313 \times 10^{9}$ & $1.16824 \times 10^{11}$ \\
\hline
\end{tabular}


From Table 2 we can conclude that the maximal error parameters for both components of the exact solution decrease approximately at an exponential rate.

Example 4. Consider a three-dimensional system of autonomous nonlinear DE with quadratic nonlinearities [33]

$$
x^{\prime}=y-a x+y z, y^{\prime}=b y-x z, z^{\prime}=c x y-d z-e x^{2},
$$

where $x, y, z \in \mathbf{R}$, and the state variables, and $a, b, c, d \in \mathbf{R}^{+}$and $e \in \mathbf{R}$ are constant parameters of the system. This system has many interesting complex behaviors and exhibits chaotic behavior over a wide range of parameters. It can show two coexisting one-wing, a single two-wing, three-and four-wing when its parameters are chosen appropriately.

When we set $a=1.6, b=3, c=8, d=11$ and $e=0.5$ the system can display two existing one-wing chaotic attractors with different initial conditions as shown in Figure 6. The right (colored in blue) and left (colored in red) attractors are constructed with initial values $(-0.5,1,0.5)$ and $(-0.5,-1,0.5)$, respectively by running NSOL for $n=10$ and step-size $h=0.02$ (left) and MATHEMATICA (right) on the interval $0 \leq t \leq 40$.

For the values of the parameters $a=8, b=4, c=8, d=11$ and $e=0.5$, the system generates two existing two-wing chaotic attractors as shown in Figure 7. Each wing of the attractor has a helical form. Running NSOL by taking $n=10$ and step-size $h=0.02$ outputs the 11 th-order numeric solutions for the system on the interval $0 \leq t \leq 40$. Two existing two-wing chaotic attractor generated by modified ADM is sketched on the left and the MATHEMATICA result is sketched on the right of Figure 7 . For each attractor we use the above initial conditions.

With the choice of the parameters $a=4.84, b=3, c=8, d=11$ and $e=0.5$ the system generates a three-wing attractor for the initial values as $x(0)=-0.5, y(0)=-1, z(0)=0.5$ as seen in Figure 8. On the left 11th-order modified ADM numeric solution for $n=10$ and step-size $h=0.02$, generated by routine NSOL and on the right MATHEMATICA numeric solution on the

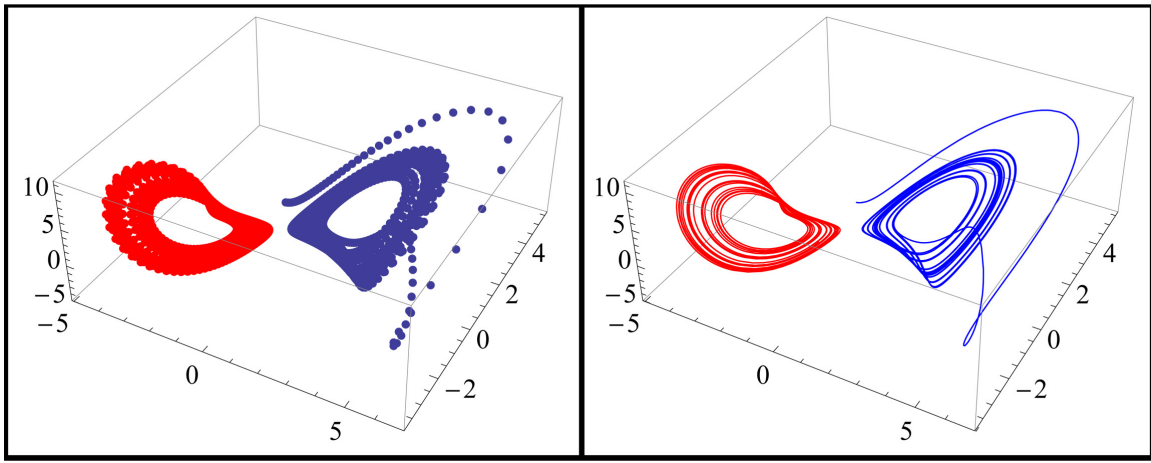

Figure 6. Phase portraits of the two coexisting one-wing chaotic attractors for $a=1.6, b=3, c=8, d=11, e=0.5$ blue line: $x(0)=-0.5, y(0)=1, z(0)=0.5$, red line: $x(0)=-0.5, y(0)=-1, z(0)=0.5$ generated by modified ADM (left) and MATHEMATICA (right). 


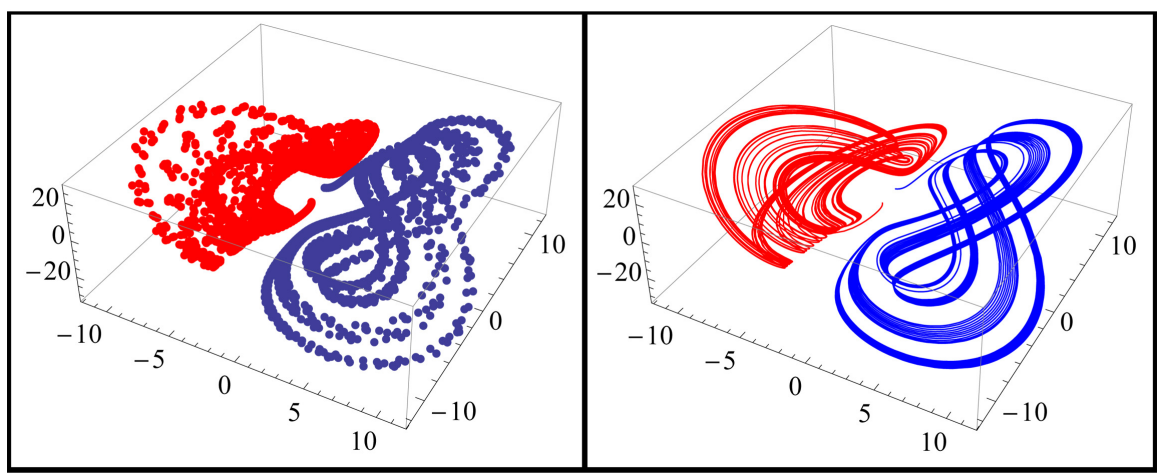

Figure 7. Phase portraits of two existing two-wing chaotic attractors for $a=8, b=4, c=8, d=11, e=0.5$ generated by modified ADM (left) and MATHEMATICA (right).

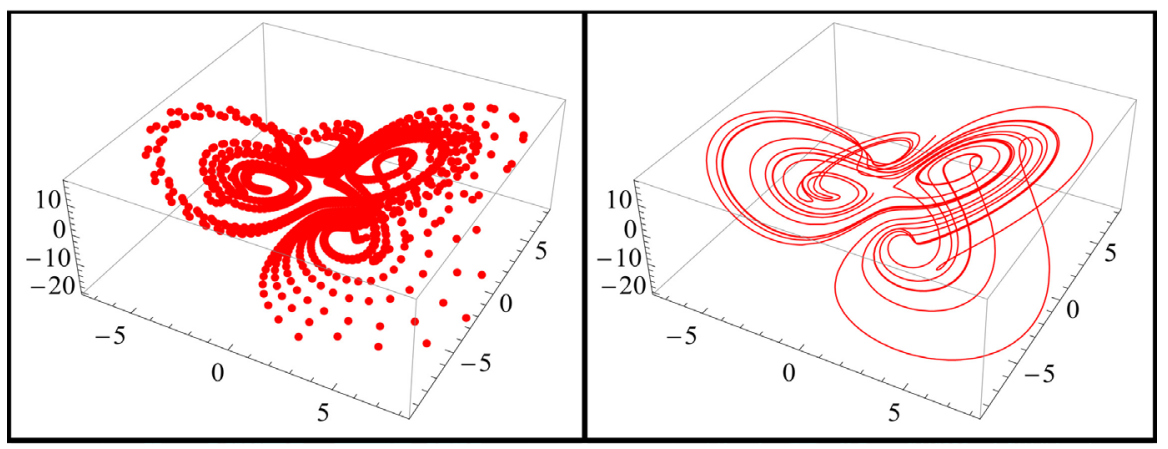

Figure 8. Phase portraits of a single three-wing chaotic attractor for $a=4.84, b=3, c=8, d=11, e=0.5$ and $x(0)=-0.5, y(0)=-1, z(0)=0.5$ generated by modified ADM (left) and MATHEMATICA (right).

interval $0 \leq t \leq 40$ are displayed.

It can also display a four-wing chaotic attractor with parameters $a=8.8, b=3, c=8, d=11$ and $e=0.5$ and initial values $x(0)=-0.5, y(0)=-1, z(0)=0.5$ as illustrated in Figure 9.

Another four-wing chaotic attractor exists for $a=9.93, b=3, c=8, d=11, e=0.5$ and for $x(0)=-0.5, y(0)=-1, z(0)=0.5$ as shown in Figure 10.

Example 5. Consider 4-dimensional system of nonlinear DE with quadratic nonlinearities [34]

$$
x^{\prime}=-y-z-w, y^{\prime}=x, z^{\prime}=a\left(y-y^{2}\right)-b z, w^{\prime}=c\left(\frac{z}{2}-z^{2}\right)-d w,
$$

where $a, b, c$, and $d$ are real parameters.

The system in (48) exhibits hypertoroidal behavior when the parameters are chosen as $a=0.2, b=d=0, c=0.04$ and the initial values are taken as $x(0)=0, y(0)=0.75, z(0)=0.2, w(0)=-0.75$ as shown in Figure 11 .

Running NSOL for $n=4$ and step-size $h=0.05$ outputs the 5th-order numeric solutions for the system on the interval $0 \leq t \leq 200$. The 3 -dimensional $x-y-z$ (top) and $y-z-W$ (bottom) projections of the modified results 


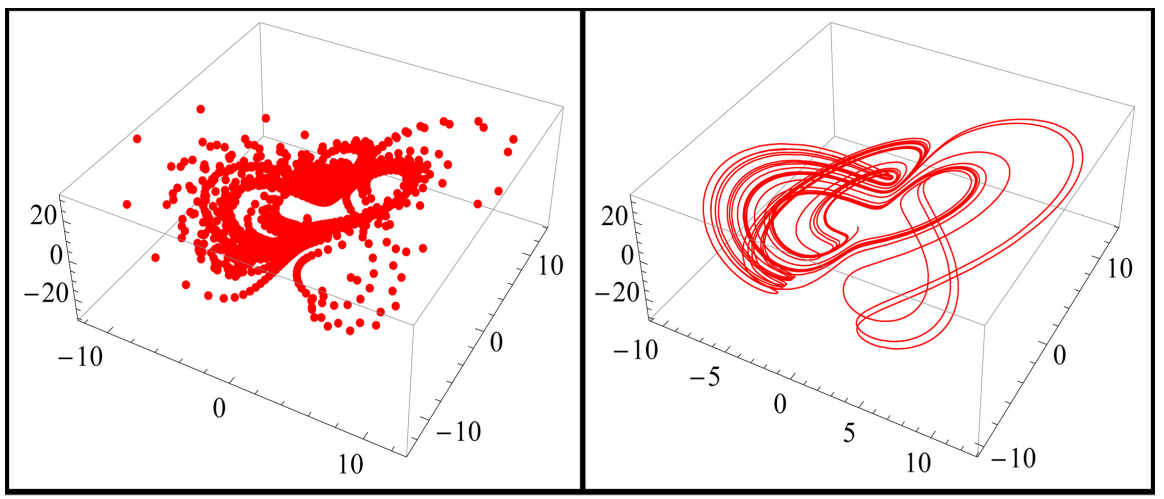

Figure 9. Phase portraits of a four-wing chaotic attractor for $a=8.8, b=3, c=8$, $d=11, e=0.5 .11$ th-order modified ADM numeric solution (left) and MATHEMATICA numeric solution (right) on the interval $0 \leq t \leq 40$.

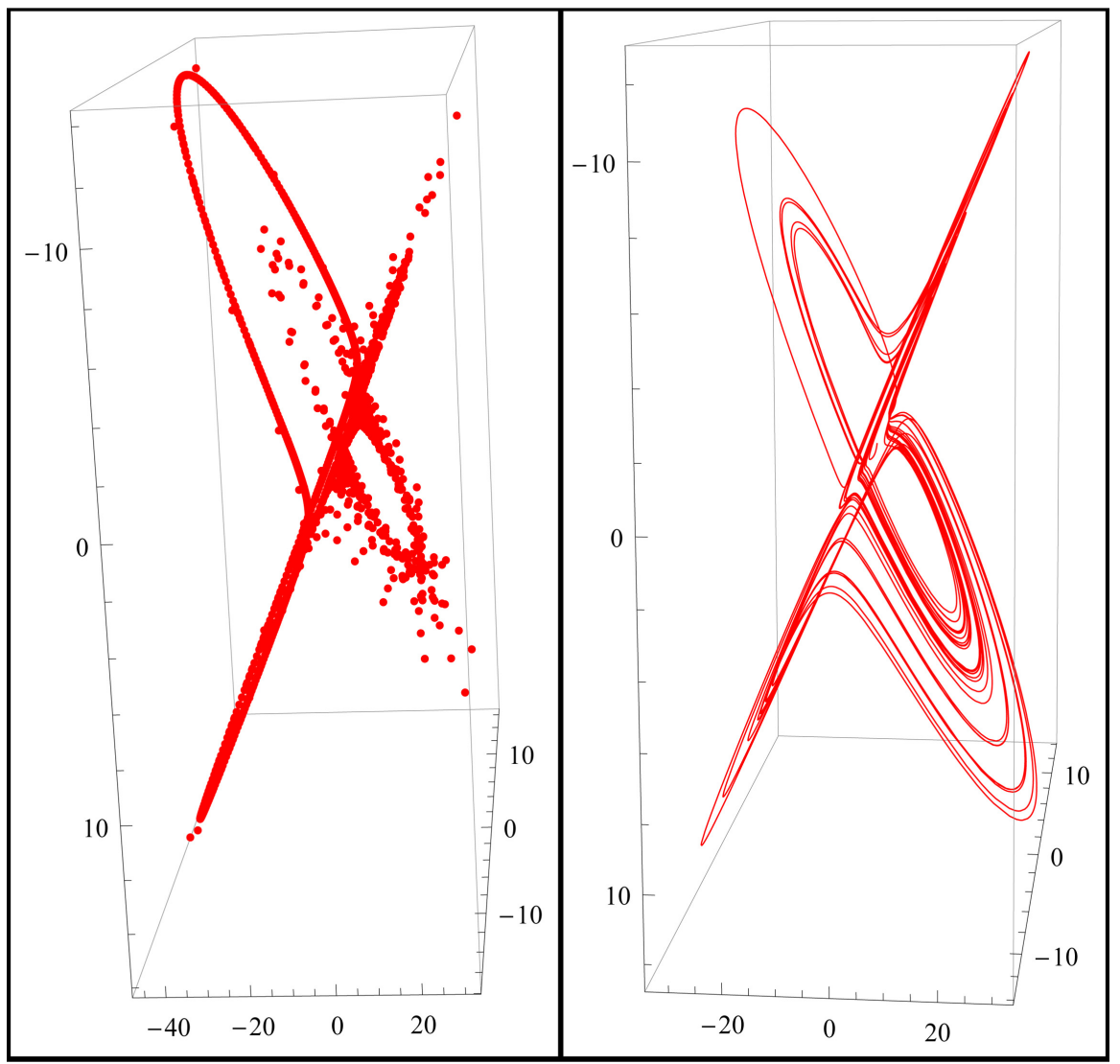

Figure 10. Phase portraits of a four-wing chaotic attractor for $a=9.93, b=3, c=8$, $d=11, e=0.5 .11$ th-order modified ADM numeric solution (left) and MATHEMATICA numeric solution (right) on the interval $0 \leq t \leq 40$.

are depicted on the left of Figure 11. To compare the results, we also calculated MATHEMATICA numeric solutions of the system are also depicted on the right of Figure 11.

Example 6. Consider the following seven-dimensional third-order hyperchaotic system [35] with cubic nonlinearity in each equation 


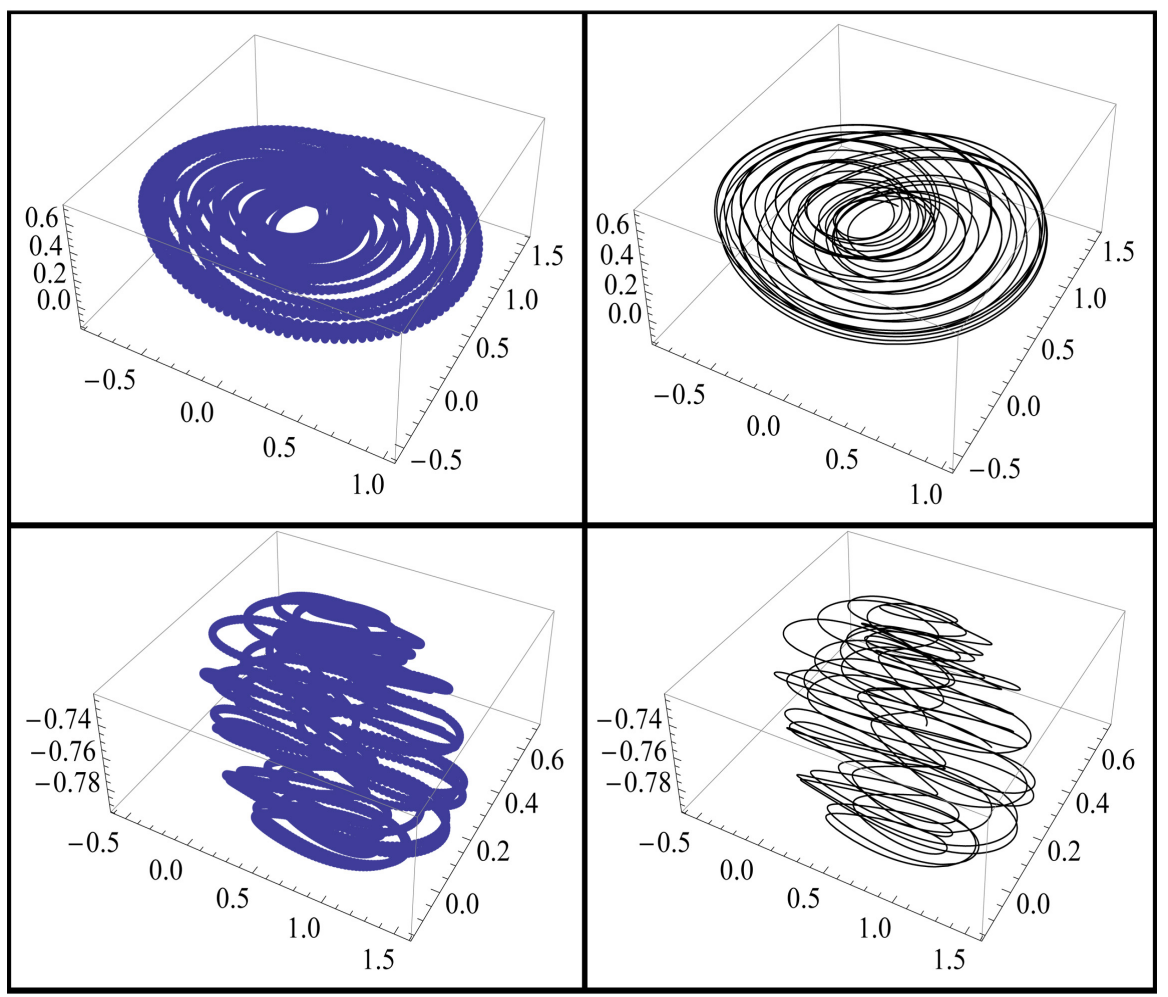

Figure 11. Hyperchaotic behavior of system (48) two different 3-dimensional $x-y-z$ and $y-Z-w$ projections of the system are shown in A and B, respectively. The parameter values are $a=0.2, b=d=0, c=0.04$ and the initial conditions are $x(0)=0, y(0)=0.75$, $z(0)=0.2, w(0)=-0.75$. On the left NSOL results and on the right MATHEMATICA results are depicted.

$$
\begin{aligned}
& x_{1}^{\prime}=-a x_{1}+a x_{5}-b x_{5} x_{6} x_{7}, \\
& x_{2}^{\prime}=-c x_{2}-d x_{6}+x_{1} x_{6} x_{7}, \\
& x_{3}^{\prime}=-a x_{3}+a x_{5}-g x_{1} x_{2} x_{7}, \\
& x_{4}^{\prime}=-a x_{4}+e x_{1}+x_{1} x_{2} x_{3}, \\
& x_{5}^{\prime}=-a x_{5}+e x_{7}-x_{2} x_{3} x_{4}, \\
& x_{6}^{\prime}=-e x_{6}+e x_{5}+x_{3} x_{4} x_{5}, \\
& x_{7}^{\prime}=-b x_{7}+f x_{2}-m x_{5} x_{6} .
\end{aligned}
$$

Since it has a chaotic attractor when we set $a=15, b=5, c=0.5, d=25$, $e=10, f=4, g=0.1, m=1.5$ and the initial conditions are taken as $x_{1}(0)=0.1, \quad x_{2}(0)=0.1, \quad x_{3}(0)=0.0, \quad x_{4}(0)=-0.2, \quad x_{5}(0)=-0.2$, $x_{6}(0)=0.0, x_{7}(0)=0.3$ we consider the numeric solution of the system (49) for those values over the interval $0 \leq t \leq 40$.

Running NSOL by taking $n=4$ and step-size $h=0.02$ generates 5 th-order numeric solutions on the interval for the system. The 2- and 3-dimensional projections of the modified results are plotted on the left of Figure 12 and Figure 13. To compare the results, we also calculated MATHEMATICA numeric solution of the system and plotted on the right of Figure 12 and Figure 13. 


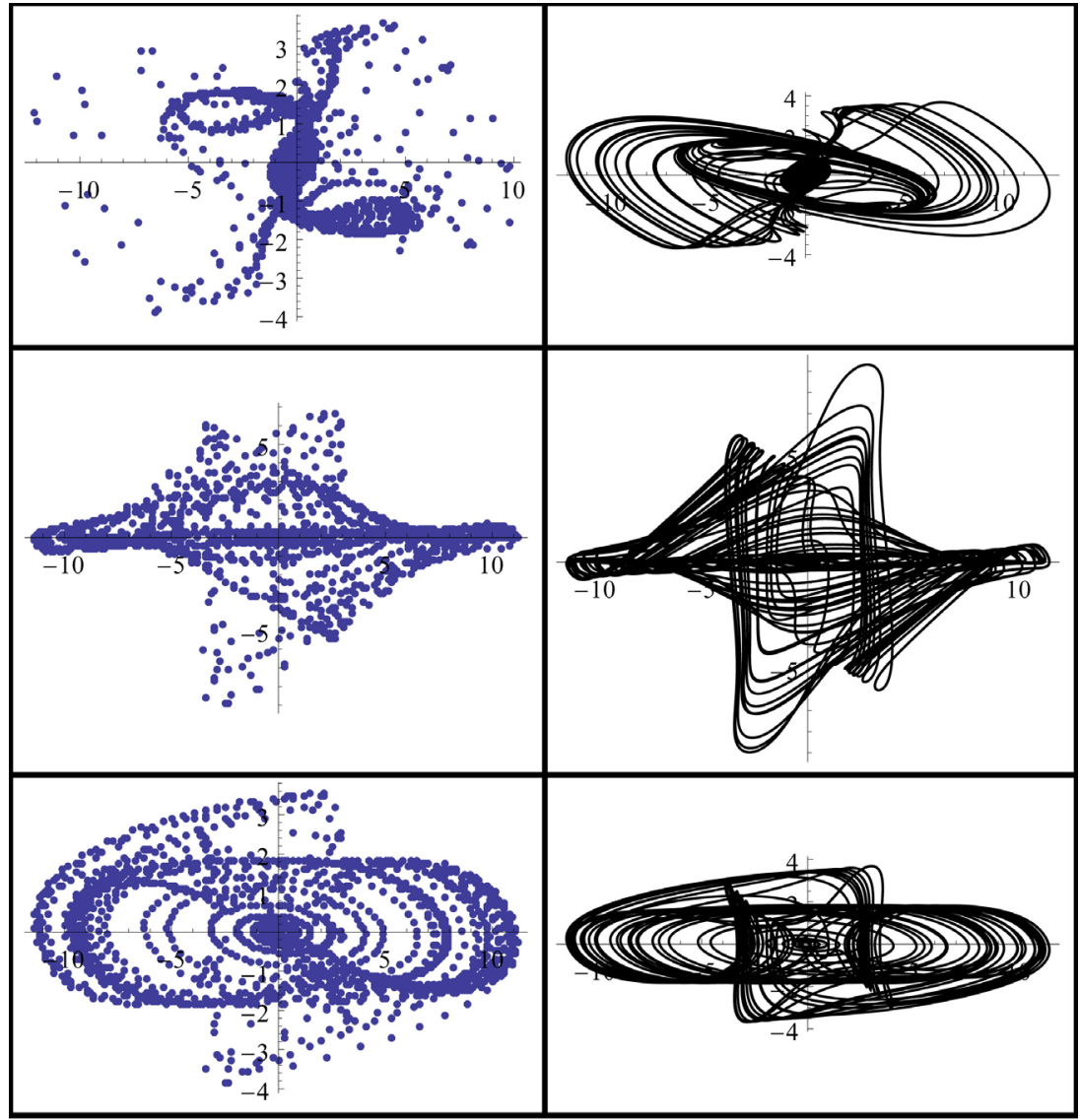

Figure 12. Two-dimensional $x_{1}-x_{6}, x_{2}-X_{4}$ and $x_{2}-x_{6}$ projections of system (49) generated by NSOL (on the left) and MATHEMATICA (on the right).

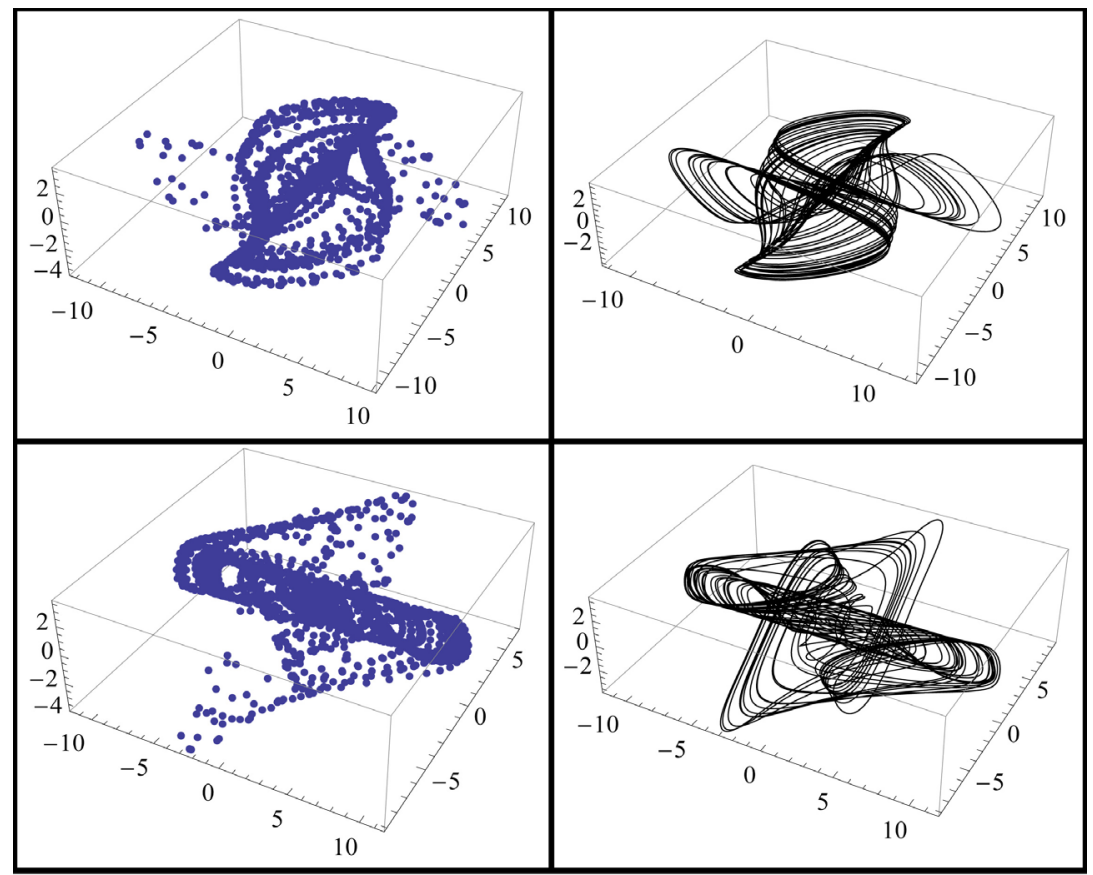

Figure 13. 3-dimensional $X_{1}-X_{2}-X_{6}$ and $X_{2}-X_{4}-x_{6}$ projections of system (49) generated by NSOL (left) and MATHEMATICA (right). 


\section{Conclusion}

In this study, we use the Duan-Rach-Wazwaz modified Adomian decomposition method for solving nonlinear IVPs of the first order nonlinear ODEs and two, three, four and seven dimensional systems of nonlinear ODEs. To show the computational accuracy of the technique we consider homogeneous and nonhomogeneous equations with variable and constant systems coefficients. In each example, the solution of the modified technique is compared with that from MATHEMATICA solution and with the exact solution if it is known. In addition, in examples 1, 2 and 3 the numeric solutions are also computed by RK4. We have seen that the modification results closely agree with MATHEMATICA and RK4 solutions and also with exact solutions, if available. However, we have obtained modified solutions over a bigger time step than MATHEMATICA and RK4 solutions. Moreover, different problems have been solved in order to confirm the robustness of the modification over a wide variety of ODEs. Therefore, it may be concluded that the method has the ability of applying all types of nonlinear ODEs provided uniqueness of the solutions.

\section{Conflicts of Interest}

The authors declare no conflicts of interest regarding the publication of this paper.

\section{References}

[1] Adomian, G. (1984) Convergent Series Solutions of Non-Linear Equations. Journal of Computational and Applied Mathematics, 11, 225-230. https://doi.org/10.1016/0377-0427(84)90022-0

[2] Adomian, G. (1988) A Review of the Decomposition Method in Applied Mathematics. Journal of Mathematical Analysis and Applications, 135, 501-544. https://doi.org/10.1016/0022-247X(88)90170-9

[3] Adomian, G. (1991) A Review of the Decomposition Method and Some Recent Results for Nonlinear Equations. Computers \& Mathematics with Applications, 21, 101-127. https://doi.org/10.1016/0898-1221(91)90220-X

[4] Adomian, G. (1994) Solving Frontier Problems of Physics: The Decomposition Method. Kluwer Academic Publishers, Boston, MA. https://doi.org/10.1007/978-94-015-8289-6

[5] Adomian, G. (1994) Solution of Physical Problems by Decomposition. Computers \& Mathematics with Applications, 27, 145-154. https://doi.org/10.1016/0898-1221(94)90132-5

[6] Adomian, G. and Rach, R. (1983) Inversion of Nonlinear Stochastic Operators. Journal of Mathematical Analysis and Applications, 91, 39-46. https://doi.org/10.1016/0022-247X(83)90090-2

[7] Adomian, G. and Rach, R. (1985) Nonlinear Plasma Response. Journal of Mathematical Analysis and Applications, 111, 114-118. https://doi.org/10.1016/0022-247X(85)90204-5

[8] Babolian, E. and Biazar, J. (2002) Solving the Problem of Biological Species Living together by Adomian Decomposition Method. Applied Mathematics and Computa- 
tion, 343, 129-339. https://doi.org/10.1016/S0096-3003(01)00043-1

[9] El-Wakil, S.A. Abdou, M.A. (2007) New Applications of Adomian Decomposition Method. Chaos, Solutions and Fractals, 33, 513-522. https://doi.org/10.1016/j.chaos.2005.12.037

[10] Aski, F.S., Nasirkhani, S.J., Mohammadian, E. and Asgari, A. (2014) Application of Adomian Decomposition Method for Micropolar Flow in a Porous Channel. Propulsion and Power Research, 3, 15-21. https://doi.org/10.1016/j.jppr.2014.01.004

[11] Adomian, G. and Rach, R. (1996) Modified Adomian Polynomials. Mathematical and Computer Modelling, 24, 39-46. https://doi.org/10.1016/S0895-7177(96)00171-9

[12] Wazwaz, A.M. (1999) Analytical Approximations and Padé Approximants for Volterra's Population Model. Applied Mathematics and Computation, 100, 13-25. https://doi.org/10.1016/S0096-3003(98)00018-6

[13] Wazwaz, A.M. (1999) The Modified Decomposition Method and Padé Approximants for Solving the Thomas-Fermi Equation. Applied Mathematics and Computation, 105, 11-19. https://doi.org/10.1016/S0096-3003(98)10090-5

[14] Wazwaz, A.M. (1999) A Reliable Modification of Adomian Decomposition Method. Applied Mathematics Computation, 102, 77-86. https://doi.org/10.1016/S0096-3003(98)10024-3

[15] Wazwaz, A.M. and El-Sayed, S.M. (2001) A New Modification of the Adomian Decomposition Method for Linear and Nonlinear Operators. Applied Mathematics and Computation, 122, 393-405. https://doi.org/10.1016/S0096-3003(00)00060-6

[16] Duan, J.S. (2010) Recurrence Triangle for Adomian Polynomials. Applied Mathematics and Computation, 216, 1235-1241. https://doi.org/10.1016/j.amc.2010.02.015

[17] Duan, J.S. (2010) An Efficient Algorithm for the Multivariable Adomian Polynomials. Applied Mathematics and Computation, 217, 2456-2467. https://doi.org/10.1016/j.amc.2010.07.046

[18] Duan, J.S. (2011) Convenient Analytic Recurrence Algorithms for the Adomian Polynomials. Applied Mathematics and Computation, 217, 6337-6348. https://doi.org/10.1016/j.amc.2011.01.007

[19] Duan, J.S. (2011) New Recurrence Algorithms for the Nonclassic Adomian Polynomials. Computers \& Mathematics with Applications, 62, 2961-2977. https://doi.org/10.1016/j.camwa.2011.07.074

[20] Duan, J.S. (2011) New Ideas for Decomposing Nonlinearities in Differential Equations. Applied Mathematics and Computation, 218, 1774-1784. https://doi.org/10.1016/j.amc.2011.06.061

[21] Duan, J.S. and Rach, R. (2011) A New Modification of the Adomian Decomposition Method for Solving Boundary Value Problems for Higher Order Nonlinear Differential Equations. Applied Mathematics and Computation, 218, 4090-4118. https://doi.org/10.1016/j.amc.2011.09.037

[22] Duan, J.S. and Rach, R. (2011) New Higher-Order Numerical One-Step Methods Based on the Adomian and the Modified Decomposition Methods. Applied Mathematics and Computation, 218, 2810-2828. https://doi.org/10.1016/j.amc.2011.08.024

[23] Duan, J.S. and Rach, R. (2012) Higher-Order Numeric Wazwaz-El-Sayed Modified Adomian Decomposition Algorithms. Computers \& Mathematics with Applications, 63, 1557-1568. https://doi.org/10.1016/j.camwa.2012.03.050 
[24] Rach, R., Wazwaz, A.M. and Duan, J.S. (2013) A Reliable Modification of the Adomian Decomposition Method for Higher-Order Nonlinear Differential Equations. Kybernetes, 42, 282-308. https://doi.org/10.1108/03684921311310611

[25] Duan, J.S., Rach, R. and Wazwaz, A.M. (2013) A New Modified ADM for Higher-Order Nonlinear Dynamical Systems. CMES, 94, 77-118.

[26] Cherruault, Y. and Adomian, G. (1993) Decomposition Methods: A New Proof of Convergence. Mathematical and Computer Modelling, 18, 103-106. https://doi.org/10.1016/0895-7177(93)90233-O

[27] Abbaoui, K. and Cherruault, Y. (1994) Convergence of Adomian's Method Applied to Differential Equations. Computers \& Mathematics with Applications, 28, 103-109. https://doi.org/10.1016/0898-1221(94)00144-8

[28] Duan, J.S., Rach, R. and Lin, S.M. (2013) Analytic Approximation of the Blow-Up Time for Nonlinear Differential Equations by the ADM-Padé Technique. Mathematical Methods in the Applied Sciences, 36, 1790-1804. https://doi.org/10.1002/mma.2725

[29] Tabet, I., Kezzar, M., Touafek, K., Bellel, N., Ghericb, S., Khelifa, A. and Adouane, M. (2015) Adomian Decomposition Method and Padé Approxiamation to Determine Fin Efficiency and Convective Straight Fins in Solar Air Collector. International Journal of Mathematical Modelling and Computations, 5, 335-346.

[30] Ismail, H.N.A., Youssef, I.K. and Rageh, T.M. (2017) Modification of Adomian Decomposition Method for Solving Fractional Riccati Differential Equation. International Advanced Research Journal in Science, Engineering and Technology, 4, 1-10.

[31] Elias, U. (2008) Qualitative Analysis of a Differential Equation of Abel. The American Mathematical Monthly, 115, 147-149.

[32] Conrad, B.P. (2010) Ordinary Differential Equations: A System Approach. https://math.temple.edu./conrad/ODEgreen.pdf https://math.temple.edu./conrad/ODEblue.pdf

[33] Dadras, S., Momeni, H.R. and Qi, G. (2010) Analysis of a New 3D Smooth Autonomous System with Different Wing Chaotic Attractors and Transient Chaos. Nonlinear Dynamics, 62, 391-405. https://doi.org/10.1007/s11071-010-9726-2

[34] Rössler, O.E. (1979) Continuous Chaos-Four Prototype Equations. Annals of the New York Academy of Sciences, 316, 376-392. https://doi.org/10.1111/j.1749-6632.1979.tb29482.x

[35] Liu, D., Zhu, J. and Sun, H. (2013) Seven-Dimensional Third-Order Hyperchaotic System and It's Applications in Image Encryption. Advanced Science and Technology Letters, 31, 119-125. 